\title{
Right Ventricular Infarction Associated with Pulmonary Embolism
}

\author{
Mohammed Al-Sadawi, Rishard Abdul, Samy I. McFarlane* \\ Department of Internal Medicine, State University of New York: Downstate Medical Center, \\ Brooklyn, New York, United States- 11203 \\ *Corresponding author: smcfarlane@downstate.edu
}

Received May 16, 2019; Revised June 25, 2019; Accepted July 05, 2019

\begin{abstract}
Background: Since the introduction of heparin as part of the management of acute coronary syndrome, the occurrence of pulmonary embolism (PE) as a complication of myocardial infarction (MI) have somewhat been unheard of. Given this rarity, its recognition is now a formidable challenge. Case Description: We present a case of a previously well 56-year-old male who presented with typical chest pain radiating to the left arm and dizziness. Initial vital signs revealed a blood pressure of $95 / 65 \mathrm{mmHg}$ and his heart rate was $42 \mathrm{bpm}$. Physical exam revealed a middle-aged male in no acute distress with cool extremities, normal heart sounds, no murmurs and flat neck veins. His chest was clear to auscultation, abdomen benign and clinically, he was euvolemic. His first electrocardiogram (EKG) was significant for bradycardia with heart block and junctional escape rhythm and ST depressions in the anteroseptal leads. Subsequent right sided EKG showed sinus bradycardia with ST segment elevations in leads II, III and aVF. Troponin I was $0.95 \mathrm{ng} / \mathrm{L}$ [normal $<0.04 \mathrm{ng} / \mathrm{L}$ ] and initial transthoracic echo (TTE) was unremarkable with an ejection fraction (EF) of 55-60\%. He was given loading doses of aspirin and clopidogrel, heparin drip initiated, and he proceeded to cardiac catheterization which revealed a $60 \%$ stenotic lesion of the mid LAD and a large filling defect with $100 \%$ stenosis of the mid RCA consistent with thrombus. Post procedure TTE revealed an EF of $40 \%$, septal and posterior hypokinesis, right ventricular regional wall motion abnormality of the basal and mid free wall with apical hypercontractility (McConnell's sign) suggestive of PE. CT pulmonary angiography revealed bilateral pulmonary emboli and anticoagulation therapy was initiated. Conclusion: This case illustrates that pulmonary embolism is a potential complication of RV infarction and its early identification is critical for implementation of anticoagulation therapy for this potentially fatal condition if left undiagnosed and untreated.
\end{abstract}

Keywords: right ventricular infarction, pulmonary embolism, coronary intervention

Cite This Article: Mohammed Al-Sadawi, Rishard Abdul, and Samy I. McFarlane, "Right Ventricular Infarction Associated with Pulmonary Embolism." American Journal of Medical Case Reports, vol. 7, no. 7 (2019): 151-157. doi: 10.12691/ajmcr-7-7-9.

\section{Introduction}

Very often, an acute pulmonary embolism may masquerade as a right ventricular myocardial infarction. However, although very rare in the era of heparin as part of acute coronary syndrome protocol, it is imperative to consider an acute pulmonary embolism as a complication of RV infarction. As there are few cases in the available literature regarding phenomenon, the pathology seems to involve the development of an intracardiac thrombus due to an akinetic myocardium with subsequent embolization in the pulmonary vasculature requiring oral anticoagulation as part of its management.

\section{Case Presentation:}

A 56 year-old male without a significant past medical history presented with central chest pain associated with near syncopal episode. He described it as substernal chest pain, radiating to his left arm associated with near syncope and dizziness. He denied orthopnea, paroxysmal nocturnal dyspnea or peripheral edema. In the ED, physical examination revealed a well developed male not in distress. He was afebrile with a blood pressure of $95 / 65$ and a heart rate of 42 . His electrocardiogram showed bradycardia with heart block and junctional escape rhythm with ST segment depression in anteroseptal leads. His troponin I was elevated to $0.95 \mathrm{ng} / \mathrm{L}$ [Normal $<0.04 \mathrm{ng} / \mathrm{L}]$. He was started on loading dose of aspirin and clopidogrel with heparin drip. His right sided electrocardiogram showed sinus bradycardia with ST segment elevation in leads II, III, aVF (inferior leads). Transthoracic echocardiography revealed revealed ejection fraction of 55 to $60 \%$. He was taken to cardiac catheterization which revealed a tubular $60 \%$ stenosis mid left anterior descending artery (LAD) and a large filling defect consistent with thrombus with $100 \%$ stenosis of mid right coronary artery (RCA) with failed thrombectomy with balloon angioplasty (Figure 4, 
Figure 5, Figure 6). His blood pressure dropped after cardiac catheterization, and hence he was started on intravenous fluids and dopamine drip. His blood pressure improved within 24 hours and dopamine drip was stopped. He was sent for post procedure transthoracic echocardiography which revealed septal and posterior hypokinesis with ejection fraction estimated to be $40 \%$. In addition, right ventricular regional wall motion abnormality of the basal and mid free wall with apical hypercontractility (McConnell's sign) suggesting pulmonary embolism (Figure 7, Figure 8). CT pulmonary angiography showed revealed filling defects of pulmonary artery branches supplying both right and lower lobes, consistent with bilateral pulmonary emboli (Figure 9). He was started on anticoagulation. In 6 weeks follow up, transthoracic echocardiography revealed ejection fraction of $45 \%$ with normal right ventricular ejection fraction and wall mobility (Figure 10).

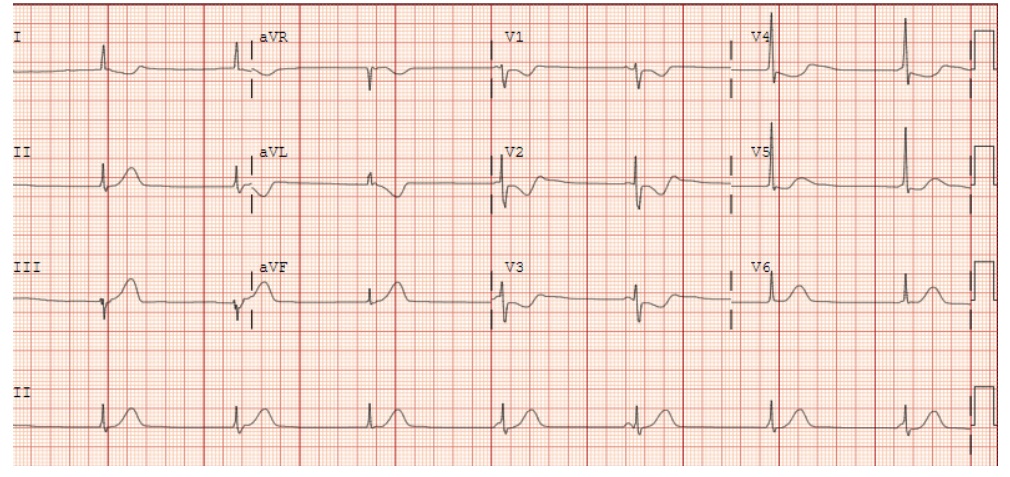

Figure 1. EKG at presentation showed bradycardia with heart block and junctional escape rhythm, ST segment depression in leads V1, V2, V3, V4 (anteroseptal leads)

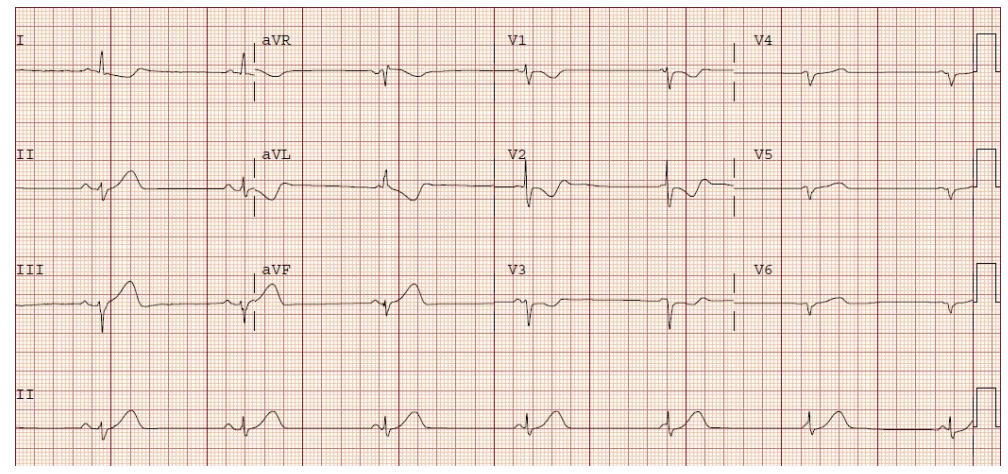

Figure 2. Right sided EKG after admission showed sinus bradycardia with ST segment elevation in leads II, III, aVF (inferior leads).

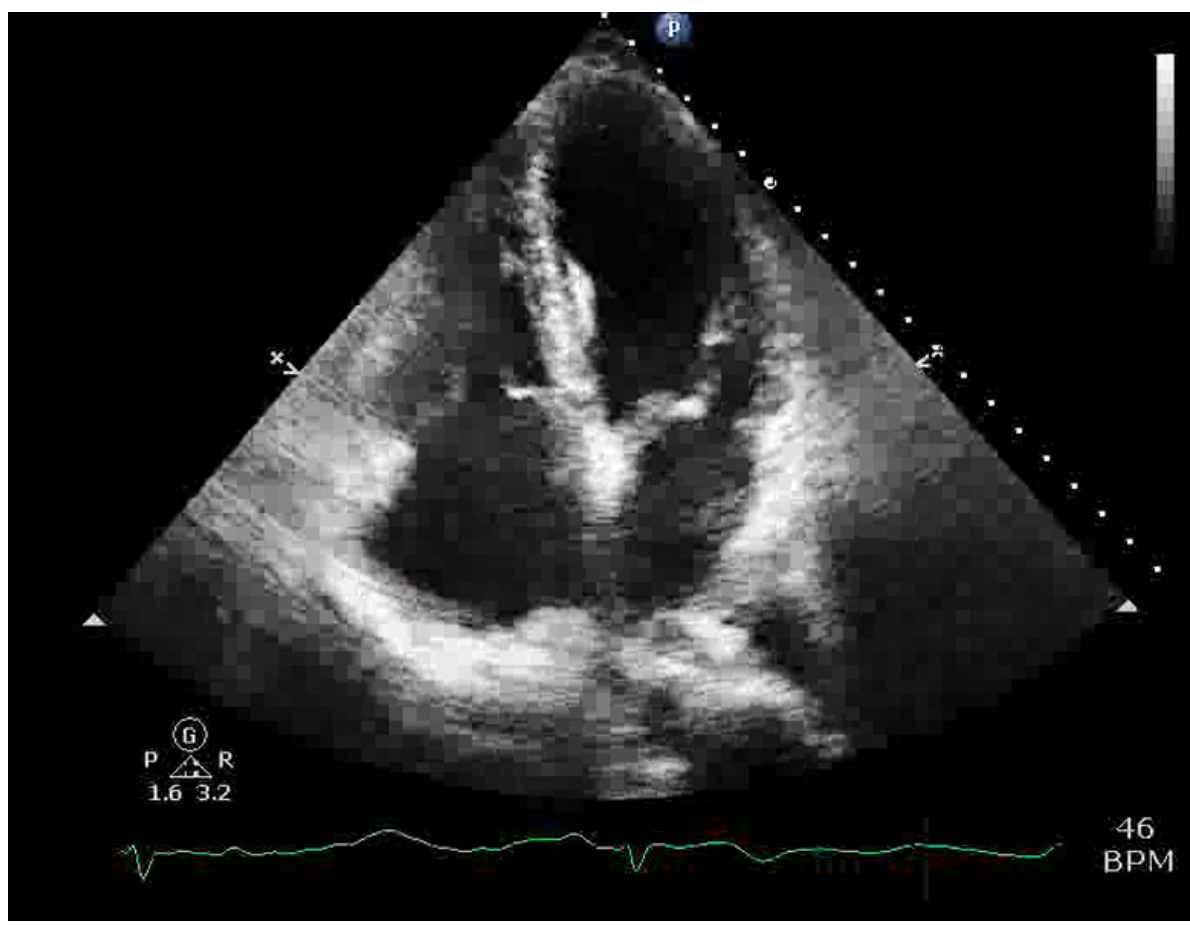

Figure 3. Pre cardiac cath transthoracic echocardiography revealed ejection fraction of 55 to $60 \%$ 


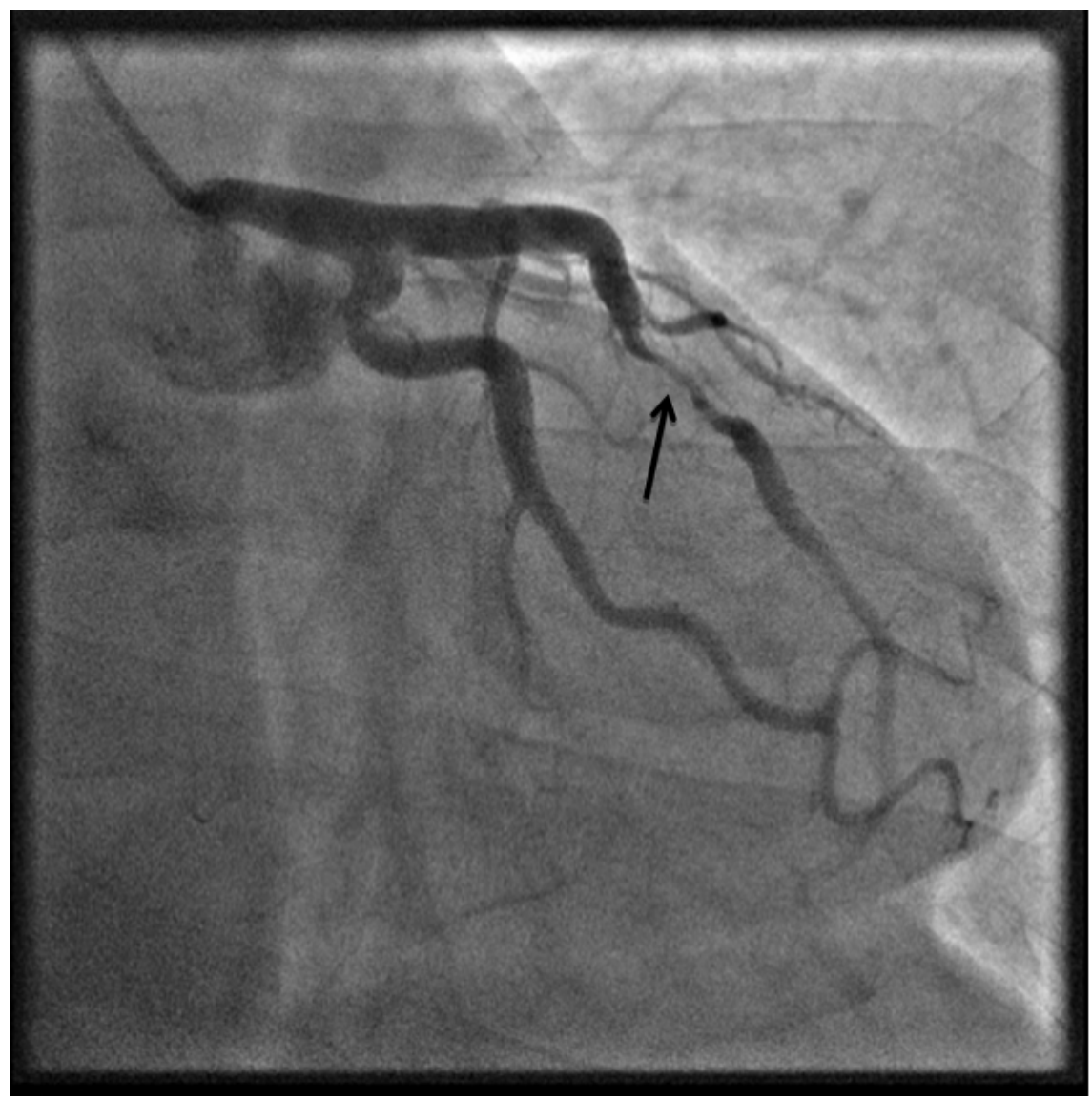

Figure 4. Cardiac catheterization revealed a tubular $60 \%$ stenosis mid left anterior descending artery (LAD) and a large filling defect consistent with thrombus with $100 \%$ stenosis of mid RCA with failed thrombectomy with balloon angioplasty

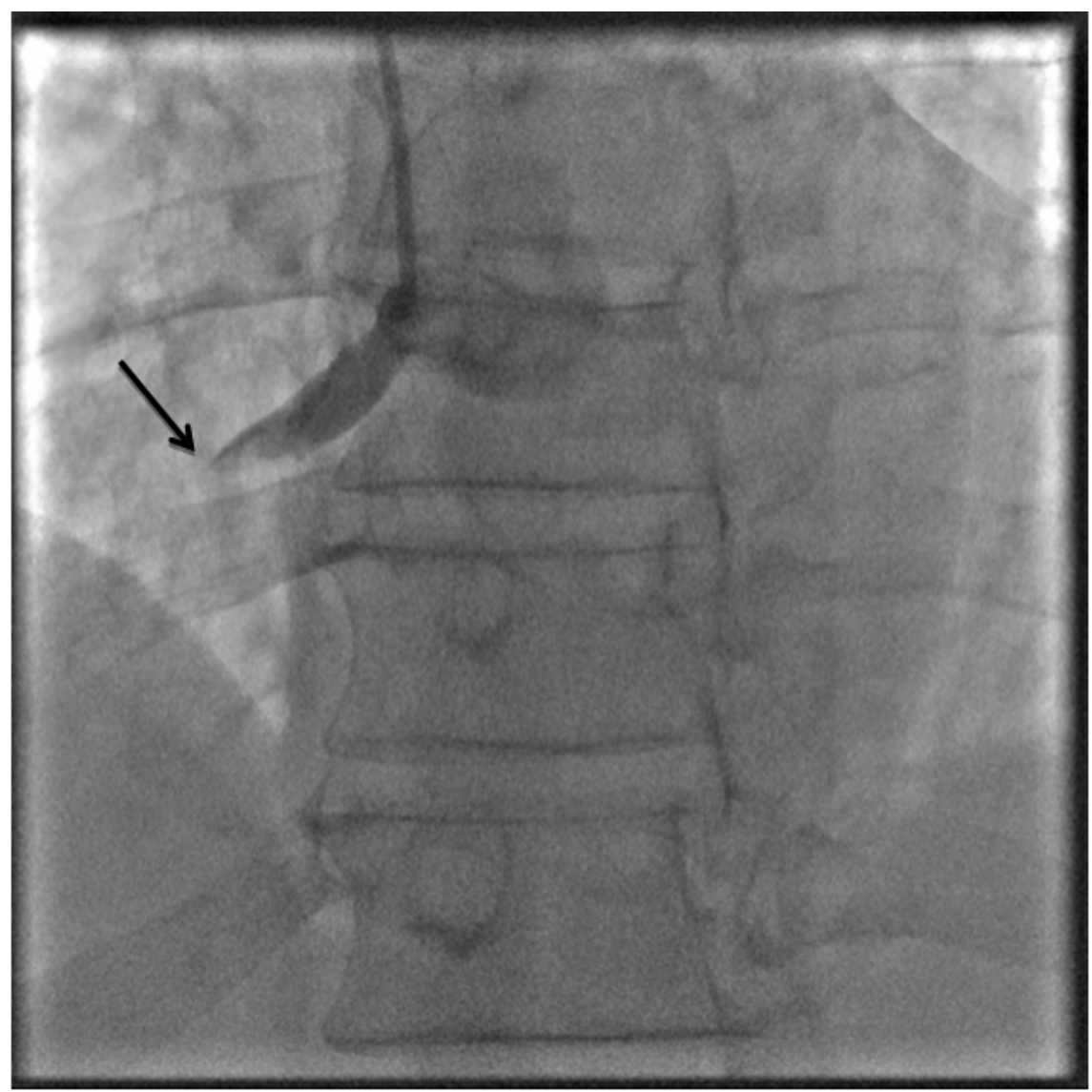

Figure 5. Cardiac catheterization revealed a tubular $60 \%$ stenosis mid left anterior descending artery (LAD) and a large filling defect consistent with thrombus with $100 \%$ stenosis of mid RCA with failed thrombectomy with balloon angioplasty 


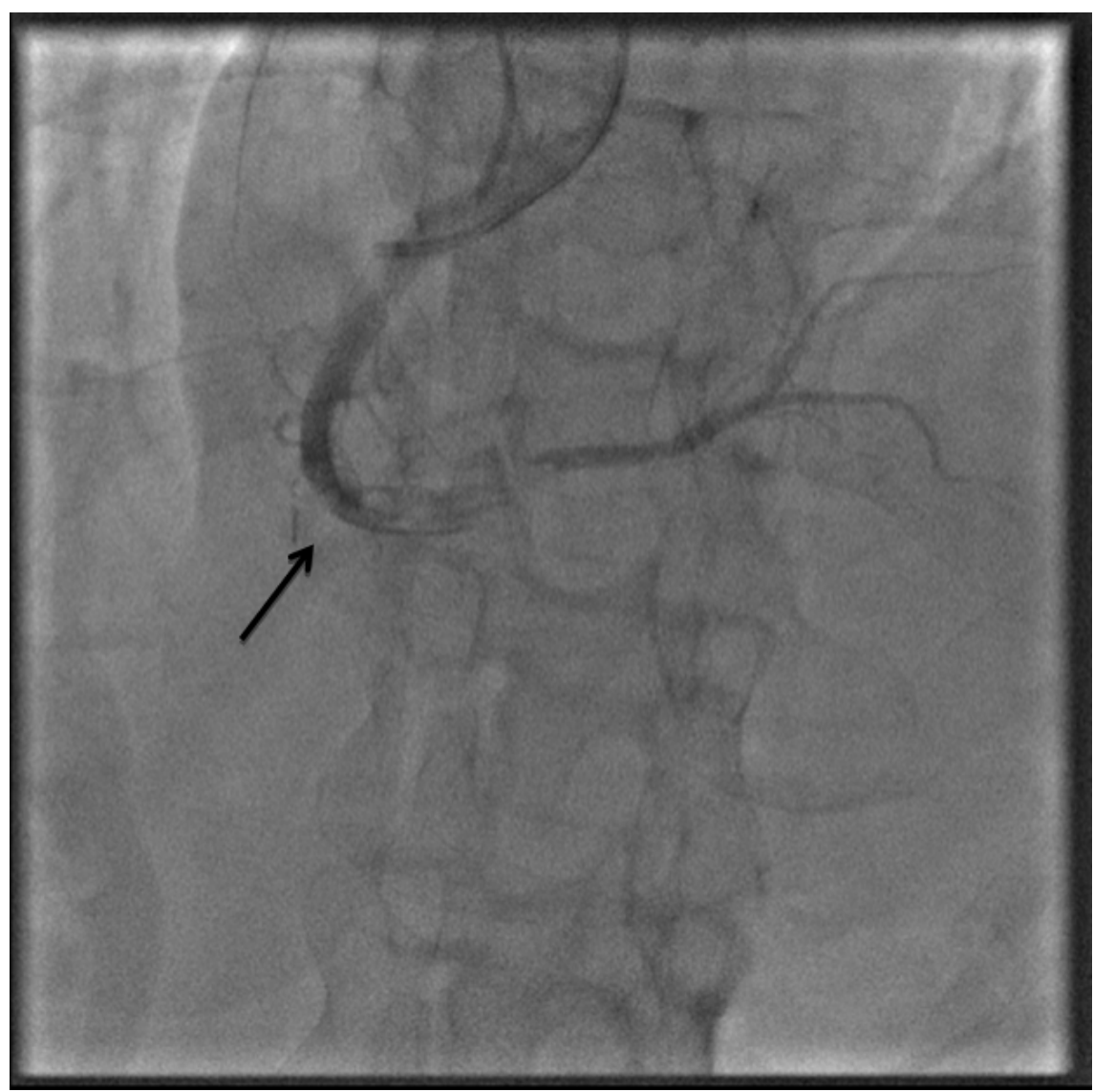

Figure 6. Cardiac catheterization revealed a tubular $60 \%$ stenosis mid left anterior descending artery (LAD) and a large filling defect consistent with thrombus with $100 \%$ stenosis of mid RCA with failed thrombectomy with balloon angioplasty

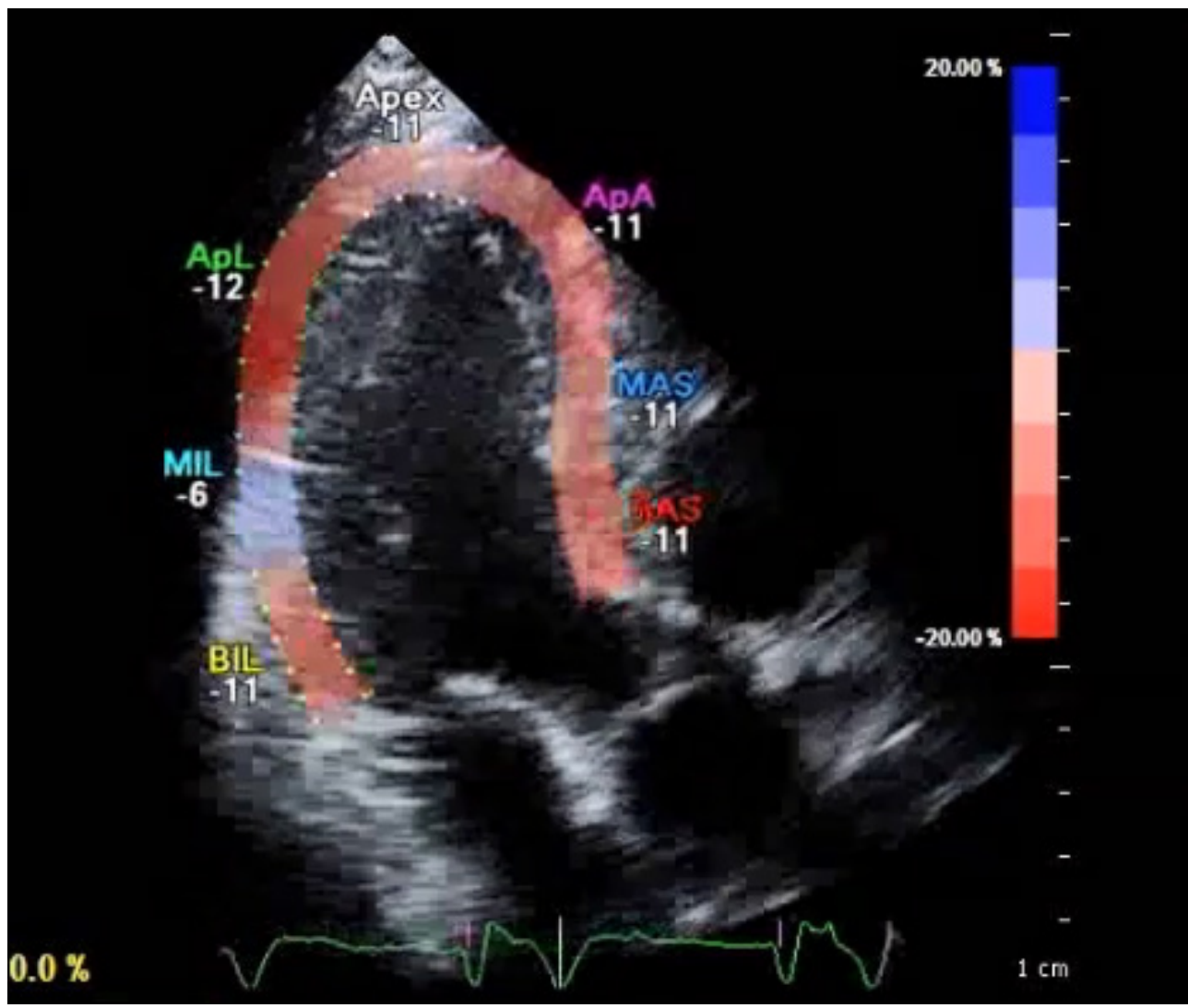

Figure 7. Post cardiac cath transthoracic echocardiography revealed septal and posterior hypokinesis with ejection fraction estimated to be $40 \%$. In addition, right ventricular regional wall motion abnormality of the basal and mid free wall with apical hypercontractility (McConnell's sign) suggesting pulmonary embolism 


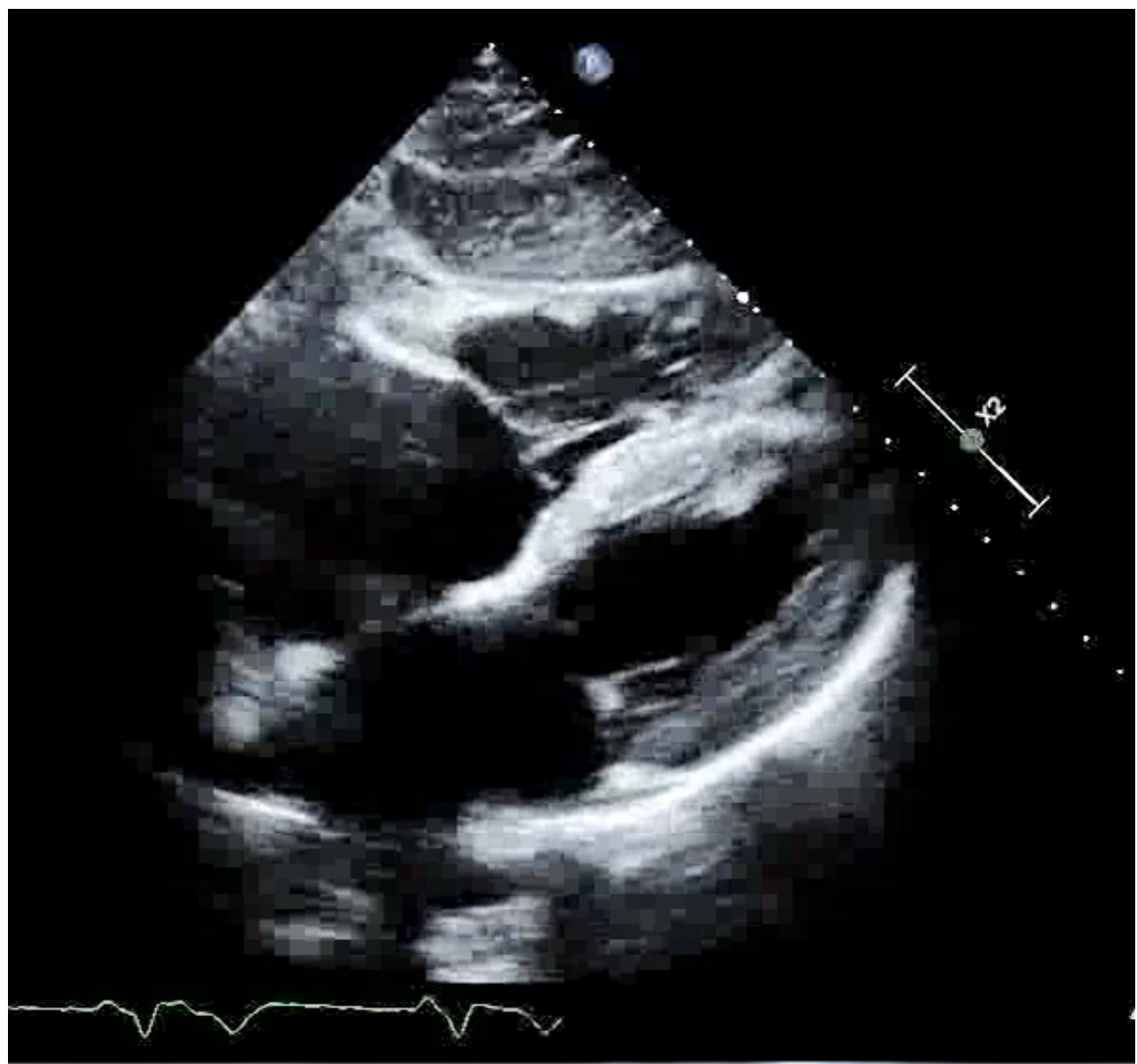

Figure 8. Post cardiac cath transthoracic echocardiography revealed septal and posterior hypokinesis with ejection fraction estimated to be $40 \%$. In addition, right ventricular regional wall motion abnormality of the basal and mid free wall with apical hypercontractility (McConnell's sign) suggesting pulmonary embolism

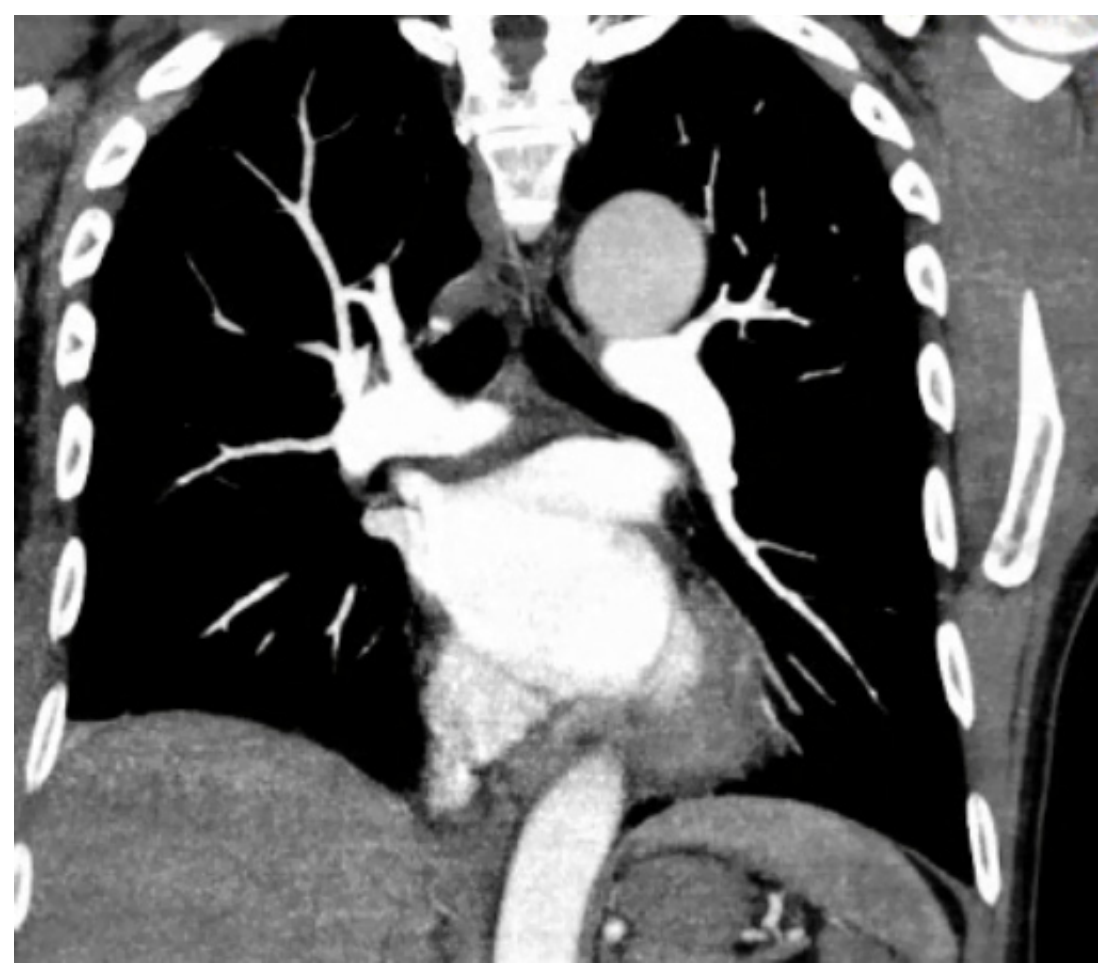

Figure 9. CT pulmonary angiography revealed filling defects of pulmonary artery branches supplying both right and lower lobes, consistent with bilateral pulmonary emboli 


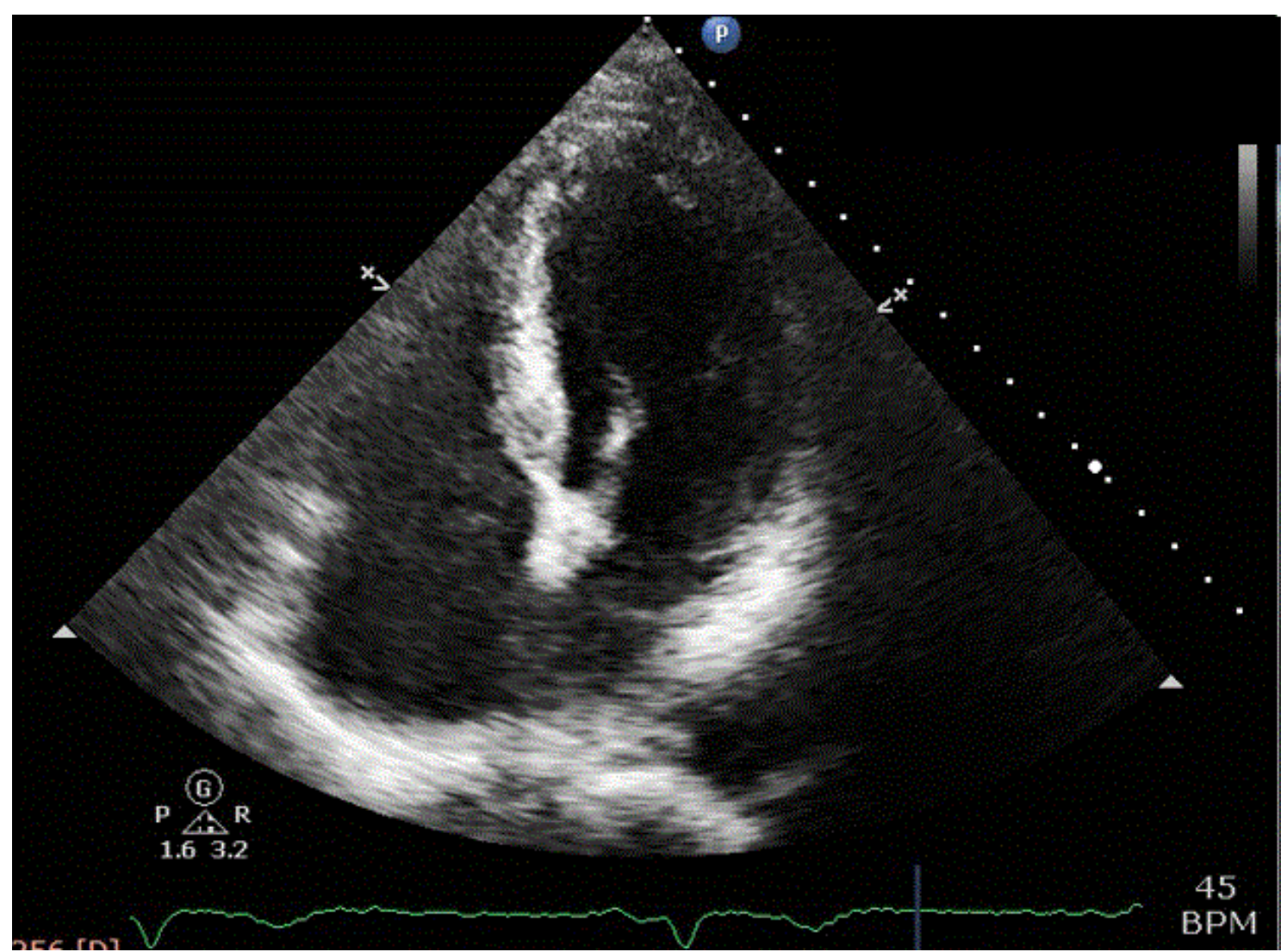

Figure 10. 6 weeks follow up transthoracic echocardiography revealed ejection fraction of $45 \%$ with normal right ventricular ejection fraction and wall mobility

\section{Discussion}

We present a case of a middle-aged man with a right ventricular (RV) myocardial infarction (MI) complicated by pulmonary embolism. While there have been numerous cases of acute pulmonary embolism (APE) masquerading as myocardial infarction, rarely are there instances of APE occurring as a complication of myocardial infarction. Our patient presented with typical clinical features of MI and the initial transthoracic echocardiogram (TTE) revealed no evidence of RV strain or pressure overload to suggest or even hint at a concomitant PE at that time. On day 3 of hospitalization, post cardiac catheterization TTE performed and interpreted by the same interpreter revealed a new McConnell's sign. While RV strain on TTE has a low sensitivity and specificity for diagnosing APE, the presence of a McConnell's sign is highly distinctive with a specificity of $94 \%$ and a negative predictive value of $96 \%$ for APE. [1,2] Thus, a CT pulmonary angiography was performed and revealed bilateral emboli in the pulmonary vasculature.

In the era of heparin as part of the management of acute coronary syndrome (ACS), APE as a complication of right ventricular MI is somewhat unheard of, except in cases of delayed presentation or delayed revascularization therapy. [3] The pathophysiology behind these cases suggest an akinetic myocardium leading to the stasis of blood followed by thrombus formation and embolization to the pulmonary arteries. Boukantar et al presented a similar case of post MI APE, however, it was in the setting of a proximal left anterior descending (LAD) infarct with delayed presentation and apical hypokinesis extending into the RV apex with resultant thrombus formation along the right septal wall. [4]

Most authors who report patients with concomitant APE and AMI describe a distinctive scenario of an APE triggering an AMI. The typical sequence of events starts with an increased pulmonary artery pressure secondary to thromboembolic phenomenon and a resultant paradoxical embolus to the coronary arteries through a patent foramen ovale (PFO). [5,6] While those patients classically have initial evidence of APE, whether it be through clinical history, physical exam, nonspecific EKG findings or TTE changes such as RV strain or bowing of the interventricular septum into the left ventricle ("D" sign) or the more specific McConnell's sign, our patient had no such suggestions. Therefore, we hypothesize that the APE occurred as a consequence of the RV infarction and not vice versa. Additionally, the coronary angiography performed on our patient revealed findings consistent with thrombus as the cause for the RCA occlusion and not an embolus as would be seen if there was paradoxical embolization in the scenario described above.

In a case series of autopsy patients published in 1952, before the use of anticoagulation in the management of AMI, pulmonary thromboembolism was considered the most common thromboembolic phenomenon in AMI. [7] However, this has been a rare sequelae of RV infarction in more recent times and have only been reported in cases with delayed presentation or delayed revascularization therapy due to the formation of $\mathrm{RV}$ thrombus and embolization. [3] In a more recent case-control cohort of 22796 autopsy patients, it was concluded that in patients who died from APE (5448/23796, 22.8\%), right sided intracardiac thrombosis was found in $354(6.5 \%)$ patients, 
and was the only detected source of PE in $220(4.0 \%)$ of those. In a sub group analysis, patients who died from ischemic heart disease had a 3.2 times higher risk of developing a right heart thrombus and was associated with a $43 \%$ PE prevalence. [8]

It is prudent to recognize APE as a complication of RV infarction since anticoagulation becomes part of the management. The American College of Chest Physicians recommends direct acting oral anticoagulants (OAC) over vitamin $\mathrm{K}$ antagonist or low molecular weight heparin for pulmonary embolism provoked by a nonsurgical transient risk factor, as in our case, and should be stopped after 3 months. [9] While there is a lack of data regarding patient's with APE secondary to RV infarction, it is recommended to add dual antiplatelet therapy to OAC among patients with STEMI and concomitant PE, with consideration of the bleeding risk. [10]

\section{Conclusion}

Albeit very rare, pulmonary embolism is important sequelae of RV infarction. While both APE and RV infarct may present with similar clinical features and can be challenging to differentiate, it is important to consider both as complications of each other in the setting of a PFO with paradoxical coronary embolization and myocardial akinesia with secondary right heart intracardiac thrombus formation with subsequent embolization.

\section{Acknowledgements}

This work is supported, in part, by the efforts of Dr. Moro O. Salifu M.D., M.P.H., M.B.A., M.A.C.P., Professor and Chairman of Medicine through NIH Grant number S21MD012474.

\section{References}

[1] McConnell, M., Solomon, S., Rayan, M., Come, P., Goldhaber, S. and Lee, R. (1996). Regional Right Ventricular Dysfunction Detected by Echocardiography in Acute Pulmonary Embolism. The American Journal of Cardiology, 78(4), pp.469-473.

[2] Oh, S., Bang, S. and Kim, M. (2015). McConnell's sign; a distinctive echocardiographic finding for diagnosing acute pulmonary embolism in emergency department. Critical Ultrasound Journal, 7(Suppl 1), p.A20.

[3] Kuno, T., Imaeda, S., Hashimoto, K., Ryuzaki, T., Saito, T., Yamazaki, H., Tabei, R., Kodaira, M., Hase, M. and Numasawa, Y. (2018). Recent Inferior Myocardial Infarction Complicated with a Right Ventricular Thrombus Detected by Three Cardiac Imaging Modalities. Internal Medicine, 57(5), pp.693-695.

[4] Boukantar, M., Lim, P. and Mitchell-Heggs, L. (2010). Right ventricular thrombus and pulmonary embolism in patient with anterior myocardial infarction. European Heart Journal, 31(23), pp. $2870-2870$

[5] Labana, S., Hussein, M., Saha, S. and Khan, M. (2017). Double Trouble: Acute Pulmonary Embolism in a Patient With Acute Inferior Wall Stemi. Chest, 152(4), p.A1024.

[6] Smith, J., Koul, S., Roijer, A., Holmqvist, J., Keussen, I., Cwikiel, W., Öhlin, B. and Erlinge, D. (2013). Acute right ventricular failure caused by concomitant coronary and pulmonary embolism: successful treatment with endovascular coronary and pulmonary thrombectomy. European Heart Journal: Acute Cardiovascular Care, 2(2), pp.131-136.

[7] Miller, R., Jordan, R., Parker, R. and Edwards, J. (1952). Thrombo-embolism in Acute and in Healed Myocardial Infarction. Circulation, 6(1), pp.7-15.

[8] Ögren, M., Bergqvist, D., Eriksson, H., Lindblad, B. and Sternby, N. (2005). Prevalence and risk of pulmonary embolism in patients with intracardiac thrombosis: a population-based study of 23 , 796 consecutive autopsies. European Heart Journal, 26(11), pp.1108-1114.

[9] Kearon, C., Akl, E., Ornelas, J., Blaivas, A., Jimenez, D., Bounameaux, H., Huisman, M., King, C., Morris, T., Sood, N., Stevens, S., Vintch, J., Wells, P., Woller, S. and Moores, L. (2016). Antithrombotic Therapy for VTE Disease. Chest, 149(2), pp.315-352.

[10] Chen, Z., Li, C., Li, Y., Tang, H., Rao, L. and Wang, M. (2019). Concomitant coronary and pulmonary embolism associated with patent foramen ovale. 